Ann. Zootech., I974, 23 (2), 213-226.

\title{
INFLUENCE DU TRAITEMENT \\ (ENSILAGE, DÉSHYDRATATION, VENTILATION) SUR LA VALEUR ALIMENTAIRE \\ DE L'ÉPI ENTIER DE MAÏS IMMATURE ET MÛR ET SUR L'UTILISATION MÉTABOLIQUE DE L'URÉE AJOUTÉE CHEZ LE JEUNE TAURILLON
}

\author{
S. Z. ZELTER, Geneviève CHARLET-LERY, G. VIROBEN et L. GUÉGUEN* \\ avec la collaboration technique de F. LE Deschault-de-Monredon, \\ Françoise Kozlowskr, Michèle Fiszlewicz et Monique Allez \\ Laboratoire de Recherches sur la conservation et l'efficacité des Aliments, \\ * Station de Recherches de Nutrition, \\ Centre national de Recherches zootechniques, I. N.R. A., \\ 78350 Jouy en Josas
}

\section{RÉSUMÉ}

On étudie, par la technique des blocs, sur de jeunes taurillons normands, âgés au départ de 6 à Io mois, les effets de l'ensilage, de la déshydratation (I00- 1 I ${ }^{\circ} \mathrm{C} \times 60 \mathrm{mn}$ ) et de la ventilation (air pulsé ambiant puis réchauffé de $+4^{\circ} \mathrm{C}$ ) d'épis entiers de maïs récoltés à un stade immature avancé (traitements $I$ et 2 ) puis mûr (traitements 3 et 4 ), supplémentés par des minéraux et de l'urée.

Les mesures effectuées sont :

a) la digestibilité de l'énergie et l'utilisation métabolique de l'azote et de certains éléments minéraux ( $\mathrm{Ca}, \mathrm{P}$ et $\mathrm{Mg}$ ) en alimentation rationnée égalisée.

b) la consommation de matière sèche et le gain de poids, en alimentation ad libitum.

La digestibilité de l'énergie et l'utilisation métabolique de l'azote ne sont aucunement influencées par la nature du traitement technologique appliqué à l'épi de maïs. La qualité exceptionnellement bonne de l'ensilage de l'épi à un stade immature relativement avancé favorise l'efficacité métabolique de l'urée ajoutée aussi bien que la déshydratation ou la ventilation. Le degré de maturité et la déshydratation abaissent légèrement la rétention de calcium et de magnésium. Les niveaux d'ingestion de matière sèche et de croissance sont les plus hauts avec le produit ventilé et les plus bas avec l'ensilage. 


\section{INTRODUC'TION}

Dans deux précédentes publications (ZELTER, CharLetT-LERY et TISSERAND, I97I ; ZELTER et CHARLE'T-LERY, I972), nous avons :

a) montré qu'en comparaison avec l'ensilage, le traitement hydrothermique. d'une céréale immature (orge, maïs) augmentait très significativement l'efficacité métabolique de l'urée ajoutée chez le jeune taurillon ;

b) admis que l'action bénéfique de ce traitement était due essentiellement au fort accroissement qu'il induit dans l'intensité de la dégradation amylolytique de l'amidon de la céréale, tandis que l'action négative de l'ensilage provenait du fait que son amidon facilement attaquable a été dégradé durant la conservation en acides organiques. Cette dégradation priverait la micropopulation ruminale d'une source particulièrement adéquate d'énergie qui lui est indispensable pour pouvoir synthétiser ses acides aminés propres à partir de l'azote 'uréique ;

c) constaté que l'action bénéfique du traitement hydrothermique ne se retrouvait pas dans le cas du grain mûr (orge) préalablement réhydraté ; bien au contraire, elle devenait même négative et ce, malgré une augmentation indiscutable de la fraction amylacée facilement dégradable, sans doute en raison de l'effet dénaturant dominant exercé par la chaleur sur les protéines de la céréale, plus spécialement sur la lysine. Nous nous sommes donc demandé s'il en était de même pour le maïs.

Pour répondre à cette question, nous avons entrepris une nouvelle expérience avec la récolte I97I d'épis entiers de maîs immature et mûr pour noter les effets de l'ensilage, de la déshydratation artificielle et de la ventilation par air pulsé sur la valeur alimentaire de cet aliment, l'efficacité azotée de l'urée ajoutée, le niveau de consommation de matière sèche et le gain de poids chez de jeunes taurillons. De plus, nous avons observé l'influence de ces régimes sur l'utilisation digestive et la rétention de $\mathrm{Ca}, \mathrm{P}$ et $\mathrm{Mg}$.

\section{MATÉRIEL, ET MÉTHODES}

\section{I. - Récolte et traitement de l'épi de mais}

L'épi entier de maïs (spathes incluses), variété INRA 258, cueilli à la main sur une même parcelle est récolté, par suite de contraintes climatiques, au stade immature avancé (le II-9-7I) avec une teneur en humidité de 50 p. Ioo puis, I 2 jours plus tard (le 23-9-7I) au stade mûr avec une humidité de 45 p. I oo.

Les traitements appliqués à cette récolte sont : ensilage et traitement hydrothermique pour l'épi immature, traitement hydrothermique et ventilation par air pulsé pour l'épi mûr.

Le maïs immature, haché à l'ensileuse à couteaux est divisé en deux lots. L'un d'eux est rebroyé dans un broyeur à marteaux et à couteaux pour disséquer tous les grains et ensilé aussitôt sous vide partiel $(40 \mathrm{~mm})$ dans des silos étanches en feuille de butyl de $4 \mathrm{~m}^{3}$ de capacité (environ 2 tonnes de matière sèche par silo); de l'urée ( $7 \mathrm{~g} / \mathrm{kgg}$ MS maïs) est ajoutée à la masse au moment de la mise en silo. Le deuxième lot de maïs immature haché est aussitôt déshydraté dans un séchoir à tablier Scolari (température de l'air au milieu du séchoir roo- I Io ${ }^{\circ} \mathrm{C}$, température de la masse 95-100 $\mathrm{C}$ ). Le temps de passage sur le tablier est de 60-75 minutes pour le grain et la rafle; il est de $45^{-5}$ o minutes pour les spathes. 
Le maïs mûr est, lui aussi, divisé en deux lots : l'un, haché à l'ensileuse à couteaux, est déshydraté exactement dans les mêmes conditions que l'immature. L'autre (environ r 6 tonnes d'épis entiers) est placé sur une aire de ventilation à fourrage, sur une hauteur de $80 \mathrm{~cm}$. La répartition de l'air pulsé par un ventilateur hélicoïdal se fait par une gaine centrale à claire-voie. Un radiateur à ailettes, placé à l'entrée de l'aire de séchage, permet, si besoin est, le réchauffement de l'air pulsé. Après 42 jours de ventilation dont les $2 \mathrm{x}$ derniers à air réchauffé de $-+4^{\circ} \mathrm{C}$, la teneur en matière sèche de la masse passe de 56 à 82,6 p. roo, le cœur de la rafle cédant très difficilement son humidité. Un broyage suivi d'une ventilation à air réchauffé durant 3 jours supplémentaires, ramène cette humidité à $\mathrm{I} 5 \mathrm{p}$. Ioo assurant ainsi la stabilisation de la masse.

\section{2. - Dispositif expérimental}

\section{a) Animaux.}

L'expérience est effectuée sur 20 taurillons normands âgés initialement de 6 à Io mois et d'un poids initial de $\mathrm{I} 60$ à $260 \mathrm{~kg}$.

Les animaux sont d'abord déparasités et vaccinés contre la fièvre aphteuse, la pasteurellose et la bronchite vermineuse et accoutumés à un régime d'orge renfermant 3 p. roo d'urée et de foin à volonté. Ils sont, ensuite, répartis en 4 groupes comparables de 5 sujets dont l'un, le plus jeune, est gardé en réserve pendant la première phase de l'expérience (mesures de digestibilité et de bilans azoté et minéral), puis intégré dans son groupe pour la seconde phase expérimentale (mesures du niveau d'ingestion de matière sèche et de croissance).

La méthode des blocs est appliquée.

Seize animaux $(4 \times 4)$ sont placés en stalles à métabolisme pour la période de digestibilité et de bilans. La durée de cette période est de I 5 jours, subdivisée en trois sous-périodes hebdomadaires successives de 5 jours. Cette période est précédée d'un mois d'accoutumance aux régimes expérimentaux.

Les animaux sont ensuite remis au sol en loges individuelles sur litière de paille et les sujets en réserve sont intégrés dans leur groupe respectif. Ils sont, après I 6 jours d'accoutumance, soumis durant 92 jours à des mesures de consommation de nourriture (alimentation ad libitum) et de croissance. Ils sont pesés 3 jours consécutifs, à l'entrée et à la sortie des périođes de bilans, et $\mathrm{I}$ fois chaque quinzaine durant la seconde phase expérimentale.

\section{b) Régimes alimentaires et modes de rationnement.}

Chacun des trois lots de maïs après déshydratation ou ventilation est broyé sur une grille de $10 \mathrm{~mm}$, additionné de paille de blé $(7.5 \mathrm{p}$. Ioo) passée sur une grille, de $5 \mathrm{~mm}$ d'urée (I $8 \mathrm{~g} / \mathrm{kg}$ de MS maïs) et d'un mélange minéral vitaminisé $(2,5 \mathrm{p}$. Ioo) (1), puis homogénéisé et pressé en bouchons de $7 \mathrm{~mm}$ de diamètre. Cette supplémentation de l'épi de maîs permet de composer des régimes équilibrés renfermant I 2 p. Ioo de cellulose Weende et théoriquement Ioo-105 g MAD/UF. Ces régimes sont donnés aux animaux tels quels accompagnés de 5 p. roo de paille grossièrement hachée, distribuée au moment des repas pour éviter des troubles de rumination.

Afin de rendre les teneurs de l'ensilage en cellulose et azote similaires à celles des régimes déshydratés, on le complète, au moment du repas, par un aliment aggloméré (I 5 p. Ioo de la MS totale de la ration) comportant 80 p. roo de paille de blé, I6,3 p. soo du même mélange minéral vitaminisé et 3.7 p. Ioo d'urée.

En expérience de digestibilité et de bilan métabolique, les animaux sont rationnés à raison de $82 \mathrm{~g}$ de $\mathrm{MS} / \mathrm{kg} \mathrm{P}^{0,75}$, ce qui, tout en évitant des refus de nourriture toujours gênants, assure théoriquement les besoins d'entretien ( 2 à $2,3 \mathrm{UF} / \mathrm{jour}$ ) et de croissance (2 à 2,6 UF/kg de gain).

En expérience de niveau de consommation et de croissance, les animaux sont alimentés ad libitum et peuvent consommer librement de la paille de litière. Chaque groupe conserve son régime de l'expérience de bilans.

Les 4 régimes expérimentaux sont indiqués ci-après :

- Mais immature ensilé + Urée (MIEU)

- Maïs immature déshydraté + Urée (MIDU)

- Maïs mûr déshydraté + Urée (MMDU)

- Maîs mûr ventilé + Urée (MMVU)

Ils sont assignés au hasard à chaque groupe.

( ${ }^{1}$ Complément ininéral en p. Ioo: $\mathrm{Ca}_{2} \mathrm{H}_{2}\left(\mathrm{PO}_{4}\right)_{2}: 23,5 ; \mathrm{CaCO}_{3}: 5 \mathrm{I}, 8 ; \mathrm{NaCl}: \mathbf{1} 8 ; \mathrm{S}: 5 ; \mathrm{ZnSO}_{4}, 7 \mathrm{H}_{2} \mathrm{O}:$ I $2 ; \mathrm{MnSO}_{4}, 7 \mathrm{H}_{2} \mathrm{O}: 0,4 ; \mathrm{CuSO}_{4}, 5 \mathrm{H}_{2} \mathrm{O}: 0, \mathrm{I} ; \mathrm{CoSO}_{4}, 7 \mathrm{H}_{2} \mathrm{O}: 0,0 \mathrm{I}$. 
c) Collecte, conservation et analyse des ingesta et excreta.

Les techniques utilisées sont décrites en détail dans nos deux articles précédents (ZELTER et al., 1971 et ZeLTer et ChARLET-LeRY, 1972).

Les dosages de $\mathrm{Ca}, \mathrm{P}$ et $\mathrm{Mg}$ sont effectués comme suit : les échantillons d'aliments, de fèces et d'urine sont minéralisés au four à $53^{\circ} \mathrm{C}$ durant 8 heures. Les cendres sont reprises sur un bain de sable par $\mathrm{HCl}$ pur, puis par $\mathrm{HNO}_{3}$ à Io $\mathrm{p}$. Ioo. Après filtration et dilution convenables, le phosphore est dosé à l'aide de la méthode colorimétrique au phospho-vanado-molybdate d'ammonium (réaction de Misson), le calcium par photométrie de flamme (appareil Eppendorf) et le magnésium par spectrométrie d'absorption atomique (appareil Hilger et Watts).

\section{RÉSULTATS}

Les résultats sont interprétés au moyen du test de Duncan.

La conservation par ensilage abaisse fortement la teneur du maiss immature en amidon total, alors que le traitement hydrothermique ou de ventilation l'augmentent Le phénomène est même fortement amplifié pour l'amidon facilement dégradable par l' $\alpha$-amylase comme l'illustrent les données du tableau I; il s'ensuit un rapport amidon facilement attaquable/urée nettement moins élevé et moins favorable dans le produit ensilé que dans les produits déshydratés.

TABLEAU I

Caractéristiques de l'état de l'amidon

\begin{tabular}{|c|c|c|c|}
\hline & $\begin{array}{l}\text { Amidon total } \\
\text { (g p. } 100 \mathrm{MS} \text { ) }\end{array}$ & $\begin{array}{c}\text { Amidon } \\
\text { facilement at taquable } \\
\text { à l' } \alpha \text {-amylase } \\
(\mathrm{g} / \mathrm{kg} \text { MS })\end{array}$ & $\begin{array}{c}\text { Rapport amidon } \\
\text { facilement attaquable/ } \\
\text { urée incorporée } \\
\text { dans la ration }\end{array}$ \\
\hline \multicolumn{4}{|l|}{ Maïs immature } \\
\hline avant traitement $\ldots \ldots \ldots$ & 48,4 & 119,0 & - \\
\hline après ensilage $\ldots \ldots \ldots \ldots \ldots \ldots$ & 30,9 & 56,0 & 2,9 \\
\hline après déshydratation...$\ldots \ldots \ldots$ & 49,4 & 178,0 & 9,9 \\
\hline \multicolumn{4}{|l|}{ Maïs mûr } \\
\hline avant traitement $\ldots \ldots \ldots \ldots \ldots$ & 49,3 & 104,0 & - \\
\hline après ventilation $\ldots \ldots \ldots \ldots \ldots$ & 58,9 & 130,0 & 7,2 \\
\hline après déshydratation $\ldots \ldots \ldots \ldots$ & 55,0 & 151,0 & 8,4 \\
\hline
\end{tabular}

La richesse en acides aminés et en 1ysine disponible est indiquée dans le tableau 2 d'où il ne ressort aucun effet particulier des traitements expérimentés.

La qualité de l'ensilage d'épis de maïs immature est exceptionnellement bonne comme le montrent les valeurs du tableau 3 : la teneur en $\mathrm{N}$ ammoniacal atteint à peine $4 \mathrm{p}$. Ioo de 1'azote total et celle en azote soluble ne dépasse pas $50 \mathrm{p}$. Ioo ; or, dans nos deux essais antérieurs (ZELTER et al., I97I) les valeurs correspondantes oscillaient entre Io,7 et I3,2 p. Ioo pour l'azote ammoniacal et entre 62,8 et $79 \mathrm{p}$. IOO 
TABIEAU 2

a) Influence des traitements étudiés sur la composition en acides aminés du mais (en $\mathrm{g}$ pour $\mathrm{I} 6 \mathrm{~g} \mathrm{~N}$ )

\begin{tabular}{|c|c|c|c|c|c|c|}
\hline & $\begin{array}{c}\text { Maîs } \\
\text { mûr } \\
\text { à la récolte }\end{array}$ & $\begin{array}{c}\text { Maïs } \\
\text { mûr } \\
\text { ventilé }\end{array}$ & $\begin{array}{c}\text { Maïs } \\
\text { mûr } \\
\text { déshydraté }\end{array}$ & $\begin{array}{l}\text { Maïs } \\
\text { immature } \\
\text { à la récolte }\end{array}$ & $\begin{array}{c}\text { Maïs }\left({ }^{1}\right) \\
\text { immature } \\
\text { ensilé + urée }\end{array}$ & $\begin{array}{c}\text { Mais } \\
\text { immature } \\
\text { déshydraté }\end{array}$ \\
\hline Acide aspartique ... & 6,9 & 6,7 & 6,9 & 6,5 & 4,3 & 6,7 \\
\hline Thréonine ...... & 4,2 & 4,0 & 4,1 & 4,1 & 2,5 & 4,4 \\
\hline Sérine $\ldots \ldots \ldots \ldots$ & 4,8 & 4,7 & 4,9 & 4,7 & 2,7 & 4,9 \\
\hline Acide glutamique .. & 18,4 & 19,4 & 19,6 & 17,9 & 10,6 & 20,4 \\
\hline Proline $\ldots \ldots \ldots \ldots$ & 8,9 & 8,7 & 9,3 & 8,6 & 5,4 & 7,3 \\
\hline Glycine $\ldots \ldots \ldots$ & 3,9 & 3,7 & 3,8 & 4,0 & 2,5 & 3,5 \\
\hline Alanine ......... & 7,7 & 7,7 & 8,0 & 7,7 & 4,6 & 7,8 \\
\hline Valine $\ldots . . . \ldots$ & 4,8 & 4,7 & 4,7 & 4,7 & 3,0 & 4,7 \\
\hline Isoleucine $\ldots \ldots \ldots$ & 3,5 & 3,6 & 3,8 & 3,7 & 2,3 & 3,7 \\
\hline Leucine ........ & 13,1 & 13,7 & 14,2 & 13,0 & 7,6 & 14,2 \\
\hline Tyrosine ........ & 4,0 & 4,3 & 4,3 & 4,1 & 2,1 & 4,3 \\
\hline Phénylalanine .... & 4,9 & 5,1 & 5,1 & 4,9 & 2,9 & 5,2 \\
\hline Lysine . . . . . & 3,1 & 2,9 & 2,7 & 2,8 & $2,8\left({ }^{2}\right)$ & 2,6 \\
\hline Histidine $\ldots$. & 2,4 & 2,7 & 2,6 & 2,3 & 1,6 & 2,6 \\
\hline Arginine $\ldots \ldots \ldots$ & 4,6 & 4,7 & 4,7 & 5,8 & 2,0 & 4,5 \\
\hline
\end{tabular}

(1) L'adjonction d'urée (41,5 p. 100 de $\mathrm{N}$ total) ayant eu lieu au moment de la mise en silo, les teneurs en acides aminés rapportées à l'azote total apparaissent obligatoirement plus faibles que celles des autres matières premières.

(2) Lysine + Ornithine.

b) Influence du mode de séchage sur la disponibilité de la lysine (en g/roo g MS)

\begin{tabular}{|c|c|c|c|c|c|}
\hline & $\begin{array}{c}\text { Maïs } \\
\text { mûr } \\
\text { à la récolte }\end{array}$ & $\begin{array}{c}\text { Maïs } \\
\text { mûr } \\
\text { ventile }\end{array}$ & $\begin{array}{c}\text { Maïs } \\
\text { mûr } \\
\text { déshydraté }\end{array}$ & $\begin{array}{c}\text { Maïs } \\
\text { immature } \\
\text { à la récolte }\end{array}$ & $\begin{array}{c}\text { Maìs } \\
\text { immature } \\
\text { déshydrate }\end{array}$ \\
\hline Lysine totale $\ldots \ldots \ldots \ldots \ldots$ & 0,257 & 0,273 & 0,233 & 0,225 & 0,228 \\
\hline Lysine indisponible $\ldots . . .$. & 0,020 & 0,012 & 0,022 & 0,024 & 0,018 \\
\hline Lysine disponible & 0,237 & 0,261 & 0,211 & 0,201 & 0,210 \\
\hline Taux de disponibilité (p. 100) ... & 92 & 96 & 90 & 90 & 92 \\
\hline
\end{tabular}


pour l'azote soluble total. De même, la richesse en amidon résiduel facilement attaquable est, dans cette expérience, de loin plus forte $(56 \mathrm{~g} / \mathrm{kg} \mathrm{MS})$ que celle des mais immatures des deux précédents essais où l'on a noté de très faibles valeurs : 9,5 et I6 $\mathrm{g} / \mathrm{kg} \mathrm{MS}$.

\section{TABLEAU 3}

Qualité de l'ensilage de maïs immature

\begin{tabular}{|c|c|}
\hline $\mathrm{pH}$ & 3,93 \\
\hline $\mathrm{N}-\mathrm{NH}_{3}$ p. $100 \mathrm{~N}$ total $\ldots \ldots \ldots \ldots \ldots$ & 3,8 \\
\hline Acide lactique $(\mathbf{1}) \ldots \ldots \ldots \ldots \ldots$ & 14,9 \\
\hline Acide acétique ${ }^{1}$ ) $\ldots \ldots \ldots \ldots \ldots$ & 3,76 \\
\hline Acide propionique (1) . & traces \\
\hline Acide butyrique ${ }^{(1)} \ldots \ldots \ldots \ldots \ldots$ & traces \\
\hline $\mathrm{N}$ soluble p. $100 \mathrm{~N}$ total $\ldots \ldots \ldots \ldots \ldots$ & 50,6 \\
\hline
\end{tabular}

(1) Pour 1000 g brut.

Le tableau 4 donne la composition des rations effectivement consommées. Cette composition concerne les régimes totaux dont $85 \mathrm{p}$. Ioo de matière sèche sont apportés par 1'épi de mais. On peut constater qu'elles sont pratiquement identiques dans tous les régimes à l'exception de celui à base de maïs immature ensilé, dont la teneur en azote uréique est sensiblement plus élevée $(4 \mathrm{I}, 5 \mathrm{p}$. Ioo $\mathrm{N}$ total) que celle des trois autres régimes expérimentaux (33,8 à 35 , I p. IO0).

\section{TABLEAU 4}

Composition des rations effectivement consommées en p. 100 de MS

\begin{tabular}{|c|c|c|c|c|c|}
\hline & $\begin{array}{c}\text { Matières } \\
\text { organiques }\end{array}$ & $\begin{array}{l}\text { Matières } \\
\text { azotées }\end{array}$ & $\begin{array}{c}\mathrm{N} \text { urée } \\
\text { p. } 100 \mathrm{~N} \text { total }\end{array}$ & $\begin{array}{l}\mathrm{N} \text { soluble } \\
\text { p. } 100 \mathrm{~N} \text { total }\end{array}$ & $\begin{array}{c}\text { Cellulose } \\
\text { Wende }\end{array}$ \\
\hline MIEU $(\mathbf{l})$ & 96,4 & 13,6 & 41,5 & 45,5 & 12,0 \\
\hline MIDU & 94,7 & 13,4 & $3: 4,7$ & 45,0 & 10,9 \\
\hline MMVU & 95,8 & 13,8 & 33,8 & 49,6 & 10,5 \\
\hline MMDU & 95,8 & 13,2 & 35,1 & 45,2 & 11,3 \\
\hline
\end{tabular}

( $\left.{ }^{1}\right) \mathrm{M}:$ mais ; $\mathrm{I}$ : immature ; $\mathrm{M}$ : mûr ; $\mathrm{E}$ : ensilé ; $\mathrm{D}$ : déshydraté ; $\mathrm{V}$ : ventilé ; $\mathrm{U}$ : urée.

\section{Digestibilité et bilan d'azote}

Le rationnement égalisé adopté a supprimé les refus de nourriture et a maintenu les niveaux de consommation de matières sèches entre 82 et $83 \mathrm{~g} / \mathrm{kg} \mathrm{P}^{0,75}$ dans les 4 groupes. 
Bien que non significativement différents d'un régime à l'autre, les coefficients d'utilisation digestive (CUD) de la matière organique sont, cependant, légèrement plus élevés d'environ 3 points pour le maïs immature ensilé. Cette différence est due essentiellement à la plus grande digestibilité de la cellulose brute de l'ensilage liée vraisemblablement à la grosseur de ses particules comparativement à la finesse de celles des produits déshydratés condensés (tabl. 5).

TABLEAU 5

Coefficients de digestibilité et valeur nutritive des régimes

\begin{tabular}{|c|c|c|c|c|c|c|c|}
\hline & $\begin{array}{l}\text { g MSI } \\
/ \mathrm{kg} \mathrm{P} \mathrm{P}^{0,75}\end{array}$ & $\begin{array}{l}\text { Matières } \\
\text { sèches }\end{array}$ & $\begin{array}{c}\text { Matières } \\
\text { organiques }\end{array}$ & $\begin{array}{l}\text { Matières } \\
\text { azotées }\end{array}$ & $\begin{array}{l}\text { Cellulose } \\
\text { Weende }\end{array}$ & $\begin{array}{c}\text { MOD } \\
(\mathrm{g} / \mathrm{kg} \mathrm{MS})\end{array}$ & $\begin{array}{c}\text { MAD } \\
\text { (g/kg MS) }\end{array}$ \\
\hline $\operatorname{MIEU}\left({ }^{1}\right)$ & 83,0 & 71,5 & $7: 3,3$ & 68,1 & $53,6^{c} c$ & 706 & 92 \\
\hline MIDU & 82,0 & 68,7 & 71,2 & 65,5 & $49,9^{b}$ & $67 / 4$ & 88 \\
\hline MMVU & 82,0 & 67,9 & 69,8 & 67,8 & $41,9^{a}$ & 668 & 93 \\
\hline MMDU & 82,5 & 67,6 & 69,8 & 66,5 & $44,2 a b$ & 669 & 88 \\
\hline & & & & & & & \\
\hline
\end{tabular}

(1) $\mathrm{M}$ : mais ; I : immature ; $\mathrm{E}$ : ensilé ; $\mathrm{D}$ : déshydraté ; $\mathrm{V}$ : ventilé ; $\mathrm{U}$ : urée.

Signification au test de Duncan des CUD cellulose $: a-b: 5$ p. $100 ; a$ et $a b-c: 1$ p. 100.

La richesse en énergie digestible (MOD g/kg MS) n'est pas influencée par le stade de maturité, alors qu'elle l'est légèrement par le traitement : l'ensilage immature renferme environ $5 \mathrm{p}$. Ioo de plus d'énergie digestible que les formes déshydratées immatures et mûres.

Il est intéressant de noter la constance remarquable de cette richesse d'une année à 1'autre, les valeurs I97 I recouvrant exactement celles enregistrées pour les récoltes I967 et I968 (ZELTER et al., r97I).

Les valeurs UF du $\mathrm{kg}$ de matière sèche $\left(^{1}\right)$ calculées théoriquement à partir de ces données, ressortent à 0,92 pour le régime à maîs immature ensilé et à $0,84-0,85$ pour les 3 autres régimes déshydratés ou ventilés et condensés.

Bien que la différence la plus élevée notée pour le bilan azoté ( $\mathrm{gN}$ bilan $/ \mathrm{kg} \mathrm{P}^{0,75}$ ) atteigne I4 p. Ioo (MIE,U-MIDU), aucun des traitements n'exerce d'influence significative sur le bilan, la rétention (CR) et 1'utilisation pratique (CUP) de l'azote. Contrairement à nos résultats antérieurs, l'utilisation métabolique de l'urée ajoutée à l'ensilage est, dans cet essai, équivalente à celle de l'urée qui supplémente les formes déshydratée ou ventilée comme le prouvent les données des tableaux 6 et 7 .

\section{Digestibilité et Rétention de Ca, $\mathrm{P}$ et $\mathrm{Mg}$}

Les résultats sont résumés dans le tableau 8 . Le coefficient d'utilisation digestive apparent (CUDa) ne tient pas compte de l'excrétion fécale endogène ; le coefficient

(1) $\mathrm{UF}=\frac{(\mathrm{MOD}+\mathrm{I}, 25 \mathrm{MGD}) 3,6-\mathrm{MS}}{\mathrm{1} 883}$. 
TABLEAU 6

Bilan de l'azote

\begin{tabular}{l|c|c|c|c|c}
\hline \hline & $\begin{array}{c}\text { Poids moyen } \\
(\mathrm{kg})\end{array}$ & $\begin{array}{c}\text { MOD } \\
/ \mathrm{kg} \mathrm{P0,75}\end{array}$ & $\begin{array}{c}\text { NDa } \\
/ \mathrm{kg} \mathrm{P0,75}\end{array}$ & $\begin{array}{c}\text { g NDa } \\
\text { p. 100 MOD }\end{array}$ & $\begin{array}{c}\text { N bilan } \\
/ \mathrm{kg} \mathrm{P}^{\mathbf{0}, 75}\end{array}$ \\
\hline & & & & \\
MIEU (1) & 261 & 58,4 & 1,22 & 2,09 & 0,51 \\
MIDU & 258 & 55,0 & $\mathbf{1 , 1 5}$ & 2,09 & 0,44 \\
MMVU & 270 & 55,0 & 1,23 & 2,23 & 0,49 \\
MMDU & 275 & 55,2 & 1,16 & 2,11 & 0,46 \\
\hline
\end{tabular}

(2) $\mathbf{M}$ : maïs ; I : immature ; $M$ : mûr ; D : déshydraté ; $V$ : ventilé ; $U$ : urée.

Aucune différence significative d'après le test de Duncan.

\section{TABLEAU 7}

Effet de la technique de conservation sur l'efficacité azotée des épis de maïs supplémentés en urée ( $\pm \mathrm{Sm}$ )

\begin{tabular}{|c|c|c|c|c|c|}
\hline \multirow{2}{*}{ Régimes } & \multirow{2}{*}{ Récolte } & \multicolumn{2}{|c|}{ Immature } & \multicolumn{2}{|c|}{ Mûr } \\
\hline & & ensilé & déshydraté & déshydraté & ventilé \\
\hline $\mathrm{CR}$ & $\begin{array}{l}1967 \\
1968 \\
1971\end{array}$ & $\begin{array}{l}22,7 \pm 5,3 \\
26,2 \pm 1,6 \\
41,5 \pm 0,9\end{array}$ & $\begin{array}{l}39,5 \pm 5,7 \\
35,2 \pm 1,0 \\
38,0 \pm 3,3\end{array}$ & $\underbrace{}_{39,7 \pm 1,3}$ & $\frac{-}{39,8 \pm 4,0}$ \\
\hline CUP & $\begin{array}{l}1967 \\
1968 \\
1971\end{array}$ & $\begin{array}{l}15,2 \pm 3,7 \\
19,5 \pm 1,3 \\
28,2 \pm 0,3\end{array}$ & $\begin{array}{l}26,4 \pm 3,5 \\
25,6 \pm 0,7 \\
24,9 \pm 2,3\end{array}$ & $\begin{array}{l}6- \\
2,4 \pm 0,8\end{array}$ & $\frac{\text { — }}{27,0 \pm 2,7}$ \\
\hline Soluble p. $100 \mathrm{~N}$ total & $\begin{array}{l}1967 \\
1968 \\
1971\end{array}$ & $\begin{array}{l}62,8 \\
79,0 \\
45,5\end{array}$ & $\begin{array}{l}45,2 \\
54,3 \\
45,0\end{array}$ & $\overline{45,2}$ & $\overline{49,6}$ \\
\hline $\begin{array}{c}\text { Amidon }\left({ }^{1}\right) \\
\text { facilement attaquable } \\
\mathbf{g} / \mathbf{k g} \text { MS de maïs }\end{array}$ & $\begin{array}{l}1967 \\
1968 \\
1971\end{array}$ & $\begin{array}{l}9,5 \\
16 \\
56\end{array}$ & $\begin{array}{r}128 \\
48 \\
178\end{array}$ & $\overrightarrow{-}$ & $\overline{130}$ \\
\hline
\end{tabular}

(1) Attaque par l' $\alpha$-amylase. 
MAÏS TRAITÉ, ET URÉE CHEZ LE TAURILLON

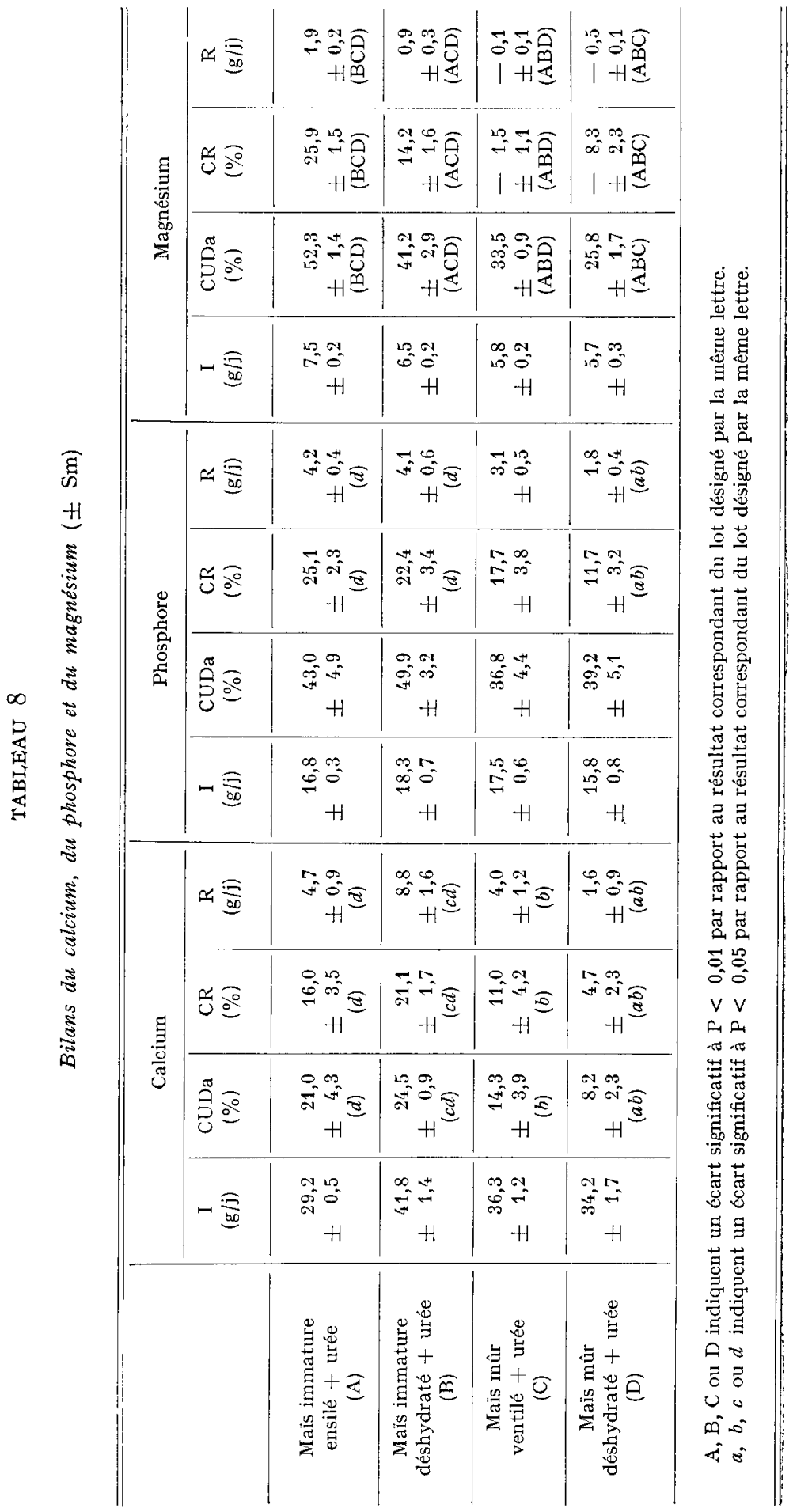


de rétention (CR) est le pourcentage de l'ingéré (I) qui est retenu (R) après déduction des pertes totales fécale et urinaire.

L'interprétation statistique (test de Duncan) fait apparaître une influence très significative $(\mathrm{P}<0, \mathrm{OI})$ de tous les traitements sur l'utilisation digestive et le bilan du magnésium : le maïs immature fournit le magnésium le plus utilisable et conduit à un bilan positif, tandis que le maîs mûr ventilé et surtout le maîs mûr déshydraté provoquent un bilan négatif du magnésium, qui semble résulter d'une faible utilisation digestive.

Les écarts observés pour le calcium sont moins significatifs $(\mathrm{P}<0,05)$ mais le maīs mûr, notamment le maïs mûr déshydraté, conduit encore à une faible utilisation et à un bilan négatif du calcium.

Pour ce qui concerne le phosphore, les différences obtenues ne sont pas significatives au niveau de l'utilisation digestive et seuls les animaux ayant reçu le maïs mûr déshydraté ont une rétention plus faible.

\section{Niveau de consommation et croissance}

Faute de réserves suffisantes d'aliments expérimentaux, il a fallu limiter la durée de cette période à 92 jours, moment où les poids moyens des animaux oscillaient entre $4 \mathrm{II}$ et $45 \mathrm{I} \mathrm{kg}$.

\section{TABLEAU 9}

Niveau de consommation et croissance

\begin{tabular}{|c|c|c|c|c|c|c|}
\hline Sm & $\begin{array}{c}\text { Poids } \\
\text { initial } \\
(\mathrm{kg})\end{array}$ & $\begin{array}{c}\text { Gain } \\
\text { moyen } \\
(\mathrm{g} / \mathrm{i} \pm \mathrm{Sm})\end{array}$ & $\begin{array}{l}\text { MISI } \\
(\mathrm{g} / \mathrm{j})\end{array}$ & $\begin{array}{l}\text { MSI/100 kg } \\
\text { de poids vif } \\
(g)\end{array}$ & $\begin{array}{c}\text { MSI } \\
/ \mathrm{kg} \mathrm{P} \mathrm{P}^{0,75} \\
(\mathrm{~g})\end{array}$ & $\begin{array}{c}\mathrm{MSI} \\
/ \mathrm{kg} \text { gain }\end{array}$ \\
\hline MIEU (1) & 291 & $\begin{array}{c}1309 \\
(973-1516) \\
\pm 103\end{array}$ & 6907 & 1961 & $84,9^{a}$ & 5,4 \\
\hline MIDU & 297 & $\begin{array}{c}1489 \\
\left(1366^{\prime}-1571\right) \\
\pm 36\end{array}$ & 7726 & 2116 & $92,5^{a}$ & 5,2 \\
\hline MMVU & $29^{\prime}$ & $\begin{array}{c}1715 \\
(1391-1981) \\
\pm 87\end{array}$ & 8661 & 2326 & $102,1^{c}$ & 5,1 \\
\hline MMDU & 301 & $\begin{array}{c}1554 \\
(1364-1853) \\
\pm 111\end{array}$ & 8121 & 2191 & $96,0^{b}$ & 5,3 \\
\hline
\end{tabular}

(1) $\mathrm{M}:$ maîs ; I : immature ; $\mathrm{M}$ : nûr ; $\mathrm{E}$ : ensilé ; $\mathrm{D}$ : déshydraté ; $\mathrm{V}$ : ventilé ; $\mathrm{U}$ : urée.

Signification des différences des niveaux d'ingestion au test de Duncan.

$a-b$ et $b-c: 5$ p. 100

$a-c: 1$ p. 100 
Les quantités de matière sèche ingérées par jour et par Ioo $\mathrm{kg}$ de poids vif ou par unité de poids métabolique $\left(\mathrm{kg} \mathrm{P}^{\mathbf{0}, 75}\right)$ indiquées dans le tableau 9 n'incluent pas la paille de litière consommée librement et non mesurée. De tous les régimes expérimentaux, celui à base d'ensilage est le moins bien consommé et celui à base de maïs ventilé l'est le mieux. La différence des niveaux d'ingestion entre ces deux régimes est de + I5,5 p. Ioo en faveur du second, et est hautement significative $(P<0,0$ r $)$. De même, l'ensilage est significativement inférieur $(\mathrm{P}<0,05)$ au maîs mûr déshydraté (-I2 p. Ioo) ; d'autre part, l'appétabilité du régime à maïs immature déshyđraté est significativement plus faible $(\mathrm{P}<0,05)$ que celle du régime à maïs mûr ventilé (一 I0,5 p. IOO).

Cependant, bien que les gains de poids journaliers assurés par chacun de ces régimes ne soient pas significativement différents, force est de constater que le groupe " ensilage " réalise la performance la plus faible (I $309 \mathrm{~g} / \mathrm{jour}$ ) soit 22,3 p. Ioo de moins que le groupe "ventilé " qui accuse le gain le plus fort (I 7 I5 g/jour). Les deux autres régimes réalisent des gains intermédiaires : I $489 \mathrm{~g} / \mathrm{jour}$ avec le mais immature déshydraté et I $554 \mathrm{~g} / \mathrm{jour}$ avec le mais déshydraté.

\section{DISCUSSION}

La constance de la composition et de la richesse en énergie digestible (MOD) du maïs immature d'une année à l'autre et l'absence de différence à cet égard entre l'épi récolté à ce stade et celui récolté au stade mûr laissent supposer que le degré de maturité est sans influence sur l'énergie digestible.

L'efficacité azotée des ensilages d'épis immatures I967 et ig68 supplémentés en urée est significativement moins bonne que celle des épis déshydratés. Dans la présente expérience, on ne note, par contre, aucune différence entre ces deux formes de présentation de l'épi immature et pas davantage entre l'épi ensilé immature et l'épi mûr déshydraté ou ventilé : les valeurs CR et CUP de l'azote sont absolument identiques à celles notées avec le maïs immature déshydraté des deux précédentes récoltes.

Apparemment donc, les données de cette expérience infirment les conclusions tirées des deux précédentes. Cependant, une analyse approfondie de l'ensemble des résultats lève cette contradiction.

En effet, il est connu que, parmi les divers facteurs qui régissent la protéo-synthèse microbienne dans le rumen à partir d'azote indifférencié, deux sont primordiaux : la disponibilité de l'énergie et la richesse en $\mathrm{N}$ soluble dans ce milieu. Dans nos premières expériences, la teneur en amidon facilement dégradable par les amylases bactériennes était beaucoup plus faible et celle en $\mathrm{N}$ soluble beaucoup plus élevée dans les ensilages que dans les formes déshydratées (tabl. Io). Cela peut donc parfaitement expliquer la moindre efficacité azotée des premiers de ces régimes. Par contre, dans la présente expérience, la teneur en $\mathrm{N}$ soluble de l'ensilage est beaucoup plus basse et identique à celle des formes déshydratées immatures ou mûtes. D'autre part, la concentration en amidon facilement dégradable est beaucoup plus forte que dans les précédents ensilages ( 48 contre $\mathrm{I} 6 \mathrm{~g} / \mathrm{kg} \mathrm{MS}$ ), elle est même légèrement supérieure 
à celle observée dans la forme déshydratée de la récolte Ig68 (56 contre $48 \mathrm{~g} / \mathrm{kg} \mathrm{MS}$ ). La conjonction de ces deux facteurs aurait, par conséquent, stimulé l'utilisation métabolique de l'azote uréique ajouté à l'ensilage; les CR des 4 régimes ne diffèrent guère entre eux dans la présente expérience : $38,0 \pm 3,3$ à $4 \mathrm{I}, 5 \pm 0,9$. Ces valeurs sont extrêmement proches de celles observées dans les 2 précédentes expériences avec les régimes immatures déshydratés supplémentés en soja ou urée $(37,4$ et 37,3$)$ et avec le régime ensilé supplémenté en soja $(37,7)$, alors que ce même ensilage supplémenté par de l'urée accuse un CR très significativement plus faible $(24,5)$ (tabl. 7).

TABLEAU IO

Composition des mais récoltés au cours des diverses années

\begin{tabular}{|c|c|c|c|c|c|c|}
\hline \multirow[b]{2}{*}{ Annẽe de récolte } & \multicolumn{3}{|c|}{$\begin{array}{l}\text { Amidon facilement attaquable } \\
\text { par l' } \alpha \text {-amylase (g/kg MS maîs) }\end{array}$} & \multicolumn{3}{|c|}{$\mathrm{N}$ soluble p. $100 \mathrm{~N}$ total } \\
\hline & 1967 & 1968 & 1971 & 1967 & 1968 & 1971 \\
\hline Imamature ensilé $\ldots \ldots \ldots$ & 10 & 16 & 56 & 63 & 79 & 45 \\
\hline Immature déshydraté ... & 128 & 48 & 178 & 45 & 54 & 45 \\
\hline Mûr dếshỵdraté $\ldots . . .$. & 一 & - & 151 & - & - & 45 \\
\hline Mûr ventilé $\ldots \ldots \ldots \ldots$ & - & $\longrightarrow$ & 130 & - & 一 & 50 \\
\hline
\end{tabular}

Nos résultats confirment nettement que si l'épi immature de maïs est ensilé à un stade de maturité suffisamment avancé, l'azote uréique qui le supplémente peut atteindre des valeurs d'efficacité azotée élevées proches de celles du tourteau de soja ; dans ce cas, le traitement hydrothermique ne présente aucun avantage. Par contre, si l'ensilage est effectué avec un épi récolté dans un état trop immature, sa pauvreté en amidon facilement dégradable et sa richesse propre excessive en $\mathrm{N}$ soluble sont des facteurs défavorables à la conversion de l'azote uréique ajouté en protéines microbiennes. JoURNET (I973) conclut dans le même sens avec des vaches laitières.

Le degré de maturité exerce aussi une action marquée sur les bilans minéraux : ceux des régimes à maïs immature, quelle que soit sa forme de présentation, sont significativement meilleurs que ceux des régimes à maïs mûr déshydraté ou ventilé. Par contre, le traitement du mais immature (ensilage ou déshydratation) n'a pas d'effet significatif.

La diffẻrence observée entre les lots immature ensilé et immature déshydraté (rétentions calciques journalières de $4,7 \mathrm{~g}$ et de $8,8 \mathrm{~g}$ ) provient surtout de la grande variabilité observée durant la première sous-période, ce qui conduit à sous-évaluer la rẻtention moyenne dans le lot ensilé et à la surévaluer dans le lot déshydraté. En considérant seulement les $2^{\text {e }}$ et $3^{\text {e }}$ sous-périodes de l'expérience, on aboutit, respectivement pour ces deux lots, à des rétentions journalières de 5,9 et de $6,5 \mathrm{~g}$ de calcium, ce qui semble plus logique compte tenu des rétentions correspondantes de phosphore (environ $4 \mathrm{~g}$ par jour).

Dans l'interprétation des résultats, il convient de distinguer d'une part, le phosphore et le magnésium, apportés par le maïs, d'autre part, le calcium, principalement 
apporté sous la forme de carbonate de calcium par le composé minéral, après le traitement de séchage ou de conservation.

La possibilité d'une influence néfaste de la déshydratation sur l'activité phytasique du grain n'est pas une explication suffisante aux variations observées. En effet, il est connu que le phosphore phytique est bien utilisé chez les ruminants et, en fait, l'absorption intestinale du phosphore semble normale dans tous les lots. Compte tenu des pertes endogènes fécales, qu'il est difficile d'estimer avec un tel régime, le CUD réel du phosphore dépasse certainement $60 \mathrm{p}$. Ioo dans tous les lots. $\mathrm{L} a$ rétention, beaucoup plus faible, observée dans le lot à maïs mûr déshydraté résulterait simplement de l'insuffisance en calcium qui ne permet pas une bonne rétention osseuse du phosphore absorbé.

Il est intéressant de noter que l'excrétion urinaire de phosphore, en général très faible chez les ruminants, devient importante lorsque le régime est riche en céréales. Ainsi, le rapport $\mathrm{P}$ fécal/P urinaire varie selon le traitement entre 2 et 3 tandis que l'élimination du calcium demeure négligeable $(\mathrm{Ca}$ fécal/Ca urinaire $=20$ à 25 ). Il est maintenant bien connu que l'augmentation de l'excrétion urinaire du phosphore se produit aux dépens de l'excrétion endogène fécale.

Indépendamment de tout traitement, le magnésium du maïs mûr est certainement moins bien absorbé que celui du maïs immature. Cela conduit à un bilan négatif de magnésium, notamment dans le cas du maïs mûr déshydraté. Bien que la teneur du régime en cet élément soit théoriquement suffisante pour couvrir les besoins des animaux, il semble nécessaire, dans le cas présent, de fournir un supplément de magnésium.

L'influence du degré de maturité et du traitement technologique de l'épi de maïs sur l'utilisation du calcium, bien que moins significative que pour le magnésium semble cependant très nette. Connaissant le poids moyen des animaux $(260 \mathrm{~kg})$ et sachant que la perte endogène fécale de calcium est constante et proche de $\mathrm{I} 8 \mathrm{mg}$ par jour et par $\mathrm{kg}$ de poids vif, il est possible de calculer le CUD réel approximatif de cet élément. Les valeurs obtenues sont normales pour le maiis immature, soit 35 à 40 p. Ioo, mais relativement faibles pour le maïs mûr, soit 25 à 30 p. Ioo.

Il est difficile de comprendre comment le degré de maturité du maïs peut influer sur l'absorption intestinale du calcium ajouté sous forme de carbonate. Bien que des modifications de la composition organique de l'aliment, notamment des teneurs en protéines, en certains aciđes aminés et en pentoses puissent modifier considérablement 1'utilisation digestive du calcium et du magnésium, il est prématuré de formuler de telles hypothèses avant d'avoir confirmé les faits observés.

L'ensilage est, indiscutablement, moins bien accepté que les produits déshydratés et, plus particulièrement, que le ventilé qui est le mieux et le plus rapidement consommé. Les CR et les CUP de l'azote étant pratiquement similaires pour tous les régimes, il est certain que la meilleure performance de croissance avec les régimes déshydratés est imputable au meilleur niveau d'ingestion de leurs matières sèches. Cela est surtout hautement significatif pour le groupe recevant le maïs ventilé dont le gain journalier de poids dépasse I 7I5 g alors que celui consommant l'ensilage n'accuse qu'un gain de I $309 \mathrm{~g}$. I1 semble donc incontestable que la présentation sous forme déshydratée et condensée accroît le niveau d'ingestion et, par conséquent, la rapidité de croissance.

Il est peu probable que les performances de croissance élevées đurant la période 
" d'appétabilité-croissance " en alimentation ad libitum soient la manifestation d'un phénomène de croissance compensatrice qui suit, généralement, une période de restriction alimentaire car, avant l'exécution des mesures, les animaux ont d'abord subi une période d'adaptation de 16 jours en alimentation ad libitum durant laquelle on a effectivement enregistré des réactions compensatrices. D'autre part, la durée de notre période expérimentale ( 92 jours) est suffisamment longue pour éliminer cet effet primaire. D'ailleurs, si l'on tient compte de la durée totale des essais (I8o jours), les gains journaliers moyens sont de I I34 g avec l'ensilage, I $368 \mathrm{~g}$ avec le mûr ventilé, I $308 \mathrm{~g}$ avec l'immature déshydraté et I $349 \mathrm{~g}$ avec le mûr déshydraté.

Il importe de souligner que les fortes performances réalisées en 92 jours par tous les groupes l'ont été sans aucun apport de tourteaux mais uniquement avec de l'épi de mais, de l'urée, de la paille et un complément minéral vitaminisé, ce qui sur le plan économique n'est pas sans intérêt vu la pénurie croissante en protéines. Toutefois, il serait intéressant de reprendre ce type d'essais sur une période de croissanceengraissement plus longue.

Reçu pour publication en novembre 1973.

\title{
SUMMARY
}

\author{
EFFECT OF THE TREATMENT (ENSILING, DEHYDRATION, VENTILATION)
}

ON THE FEEDING VALUE OF IMMATURE AND RIPE WHOLE MAIZE COBS AND ON THE METABOLIC UTILIZATION OF UREA IN YOUNG BULLS

The method of blocks is used in this experiment on young Norman bulls (initial age : 6-1o months). We study the effects of ensiling, dehydration (IOO-IIO ${ }^{\circ} \mathrm{C} \times 60 \mathrm{mn}$ ) and ventilation (circulation of surrounding air followed by $+4^{\circ} \mathrm{C}$ heating) of whole maize cobs harvested at late immaturity (treatments $I$ and 2 ) and ripe (treatments 3 and 4 ) supplemented by minerals and urea.

The following measurements are made :

a) energy digestibility and metabolic utilization of protein and of some mineral components (Ca, P, Mg) during restricted feeding,

b) dry matter intake and weight gain during ad libitum feeding.

The digestibility of the energy and metabolic utilization of the protein are not at all affected by the type of technological treatment applied to the maize cob. "The exceptionally high silage quality of the maize cobs prepared at late immaturity influence the metabolic efficiency of the urea added as the dehydration and ventilation do. The full ripeness and dehydration slightly decrease the retention of calcium and magnesium. The highest dry matter intake and growth rate are obtained with the ventilated product and the lowest values are recorded with the silage.

\section{RÉFÉRENCES BIBLIOGRAPHIQUES}

Journet M., I973. Utilisation par les vaches laitières de l'urée ajoutée à une ration d'ensilage de maïs récolté à un stade de maturité peu avancé. Ann. Zootcch., 22, i I5-II9.

Zelter S. $Z$., Charlet-Lery G., i 972. Influence du traitement hydrothermique d'une orge immature ou mûre réhydratée, sur sa valeur alimentaire et celle de l'urée ajoutée chez le jeune taurillon. Ann. Zootech., 21, 332-345.

Zelter S. Z., Charlet-Lery G., Tisserand J. L., i97i. Influence, chez le taurillon en croissance, du traitement de conservation (ensilage ou déshydratation) de la céréale immature (orge, maīs) sur sa valeur nutritive et sur l'efficacité nućtabolique de l"uréc ajoutée. Ann. Zootech., 20, 135-152. 